\title{
Comparing the performance of circulating cathodic antigen and Kato-Katz techniques in evaluating Schistosoma mansoni infection in areas with low prevalence in selected counties of Kenya: a cross- sectional study
}

\author{
Collins Okoyo* (D), Elses Simiyu, Sammy M. Njenga and Charles Mwandawiro
}

\begin{abstract}
Background: Kato-Katz technique has been the mainstay test in Schistosoma mansoni diagnosis in endemic areas. However, recent studies have documented its poor sensitivity in evaluating Schistosoma mansoni infection especially in areas with lower rates of transmission. It's the primary diagnostic tool in monitoring impact of the Kenya national school based deworming program on infection transmission, but there is need to consider a more sensitive technique as the prevalence reduces. Therefore, this study explored the relationship between results of the stool-based Kato-Katz technique with urine-based point-of-care circulating cathodic antigen (POC-CCA) test in view to inform decision-making by the program in changing from Kato-Katz to POC-CCA test.

Methods: We used two cross-sectional surveys conducted pre- and post- mass drug administration (MDA) using praziquantel in a representative random sample of children from 18 schools across 11 counties. A total of 1944 children were randomly sampled for the study. Stool and urine samples were tested for S. mansoni infection using Kato-Katz and POC-CCA methods, respectively. S. mansoni prevalence using each technique was calculated and 95\% confidence intervals obtained using binomial regression model. Specificity (Sp) and sensitivity (Sn) were determined using $2 \times 2$ contingency tables and compared using the McNemar's chi-square test.

Results: A total of 1899 and 1878 children were surveyed at pre- and post-treatment respectively. S. mansoni infection prevalence was 26.5 and $21.4 \%$ during pre- and post-treatment respectively using POC-CCA test, and 4.9 and $1.5 \%$ for pre- and post-treatment respectively using Kato-Katz technique. Taking POC-CCA as the gold standard, Kato-Katz was found to have significantly lower sensitivity both at pre- and post-treatment, $\mathrm{Sn}=12.5 \%$ and $\mathrm{Sn}=5$. $2 \%$ respectively, McNemar test $x_{m}^{2}=782.0, p<0.001$. In overall, the results showed a slight/poor agreement between the two methods, kappa index $(k)=0.11, p<0.001$, inter-rater agreement $=77.1 \%$.

Conclusions: Results showed POC-CCA technique as an effective, sensitive and accurate screening tool for Schistosoma mansoni infection in areas of low prevalence. It was up to 14-fold accurate than Kato-Katz which had extremely inadequate sensitivity. We recommend usage of POC-CCA alongside Kato-Katz examinations by Schistosomiasis control programs in low prevalence areas.
\end{abstract}

Keywords: Schistosoma mansoni, Kato-Katz, Circulating cathodic antigen

\footnotetext{
* Correspondence: comondi@kemri.org; collinsomondiokoyo@gmail.com Medical Research Institute (KEMRI), P.O. Box 54840 - 00200, Nairobi, Kenya
}

(c) The Author(s). 2018 Open Access This article is distributed under the terms of the Creative Commons Attribution 4.0 International License (http://creativecommons.org/licenses/by/4.0/), which permits unrestricted use, distribution, and reproduction in any medium, provided you give appropriate credit to the original author(s) and the source, provide a link to the Creative Commons license, and indicate if changes were made. The Creative Commons Public Domain Dedication waiver (http://creativecommons.org/publicdomain/zero/1.0/) applies to the data made available in this article, unless otherwise stated. 


\section{Background}

Existing guidelines by world health organization (WHO) for control and elimination of Schistosomiasis recommends baseline evaluations of the prevalence of Schistosome infections to inform programmatic decisions on target populations and treatment frequency within endemic areas [1]. In evaluating Schistosoma mansoni, mapping by examinations for parasite eggs in the stool using Kato-Katz thick smear microscopic technique is usually the practice [2]. This technique is usually preferred by control programmes in areas of moderate to high infection intensity and prevalence due to its ability to give both intensity and prevalence data for large sample of subjects. Due to its extensive use, it was established as part of WHO guidelines for morbidity control programmes [3]. The guideline recommends examining one stool per subject using two separate Kato-Katz slides read by two microscopists when estimating prevalence and intensity for control programmes, this is believed to provide near true prevalence estimate in high-mean intensity areas [4].

However, the Kato-Katz technique has showed day-today and intra-stool variability especially in areas with low intensity of infection and prevalence [5-7], with the cause of day-to-day variation believed to be biological [8], and the intra-stool variation attributed to sampling error due to the amount of stool evaluated [9]. This has rendered the technique less useful especially in areas with lower rates of transmission with many studies now projecting less true prevalence estimates from this technique as the population mean intensity of infection decreases [1, 10-13]. Programmes depending on single stool examination possibly lead to underestimated true infection prevalence with such observation resulting in an inaccurate allocation of control measures.

Owing to the reduced sensitivity of Kato-Katz technique in areas of low endemicity, improved diagnostic methods for accurate detection of $S$. mansoni in the at-risk populations and monitoring progress of control programmes are desirable. Several studies have now documented the availability of indirect diagnostic tests like point-of-care circulating cathodic antigen (POC-CCA) as valuable alternatives to the direct parasitological methods for diagnosis of $S$. mansoni $[6,14,15]$. This test detects Schistosome glycoprotein in the host urine after being deposited into the bloodstream by actively feeding worms and subsequently cleared from the host's kidneys [14]. The Schistosome antigens; both CCA and circulating anodic antigen (CAA) are detectable in urine of infected individuals and they act as specific markers for the presence and intensity of infection $[6,16,17]$. Studies that have been conducted in Kenya [18], Uganda [15, 19], and Ivory Coast [16] to assess the performance of CCA urine dipstick and POCCCA test among pre-school and school-aged children have both determined the usefulness of this technique in detection of S. mansoni infection in those age groups. These findings have been corroborated further by systematic reviews that have found POC-CCA to be up to 6-fold accurate than Kato-Katz technique especially in areas of low prevalence [5].

In Kenya, the ministries of health and of education began a national school-based deworming (NSBD) programme in the year 2012 in 27 counties identified as having a high prevalence of both soil-transmitted helminthes (STH) and Schistosome infections [20, 21]. According to the Kenyan National School Health Policy, treatment for both STH and Schistosome infections is administered to all school-aged children including those out of school, based on the baseline prevalence in the identified areas so as to reduce infection [21]. This national programme has been using KatoKatz technique as the primary diagnostic tool in monitoring the impact of infection transmission over a five-year period (2012-2017). Having reduced the prevalence of S. mansoni infection over the five years, and due to the growing concern over the reduced sensitivity of Kato-Katz technique, this current cross-sectional study was therefore designed to compare the performance of the stool-based Kato-Katz technique with the commercially available urine-based POC-CCA test with the view to inform decision-making by the programme in changing from Kato-Katz to POC-CCA test in its effort to control transmission of Schistosomiasis.

\section{Methods \\ Study design}

This current study was nested within the ongoing five-year monitoring and evaluation (M\&E) programme of the NSBD programme which utilized a series of pre- and post- intervention, repeat crosssectional surveys in a representative, stratified, twostage sample of schools across Kenya [20, 21]. However, our study used two cross-sectional surveys conducted during pre- and post-treatment at year five of that $M \& E$ programme. We used simple random sampling technique to randomly sample 18 schools out of the 200 schools currently being followed by the M\&E programme, the included schools across 11 counties from Coastal, Rift Valley, Nyanza and Western regions of Kenya were based on their low S. mansoni prevalence of circa $10 \%$ (using Kato-Katz technique) at fourth year of that programme [22].

In each school, 18 children ( 9 girls and 9 boys) were sampled randomly from each of the six classes; one early childhood development (ECD) class and classes 2-6 using random number tables, for a total of approximately 108 children per school. Therefore, a random sample size of 18 schools with approximate total of 108 children per school was adequate to detect a $5 \%$ change in prevalence between the two methods while assuming a power of $\beta=0.8$ and test size $\alpha=0.05$. 


\section{Data collection}

The pre-treatment surveys were conducted between 23rd January and 26th May, 2017, while post-treatment surveys were conducted between 13th March and 20th July, 2017 approximately 12-30 days after mass drug administration (MDA) to all school children by NSBD programme. Laboratory data reporting form was programmed onto android-based smart phones and used to capture data electronically into the open data kit (ODK) system [23], which included in-built data quality checks to prevent data entry errors.

\section{Survey procedures}

\section{Stool sample processing using Kato-Katz technique}

Each participating child was asked to provide stool sample which was then processed in the laboratory within $24 \mathrm{~h}$ and examined in duplicate for the presence of $S$. mansoni eggs by two different technicians using the Kato-Katz thick-smear technique and prepared using a sieve and calibrated template of $41.7 \mathrm{mg}$, any resulting discrepancies were resolved by a third senior technician. For purposes of quality control, $10 \%$ of all stool samples were randomly re-examined by a senior technologist. The readings were recorded as number of eggs per gram of stool (EPG) for each child [24]. However, for purposes of this study, the $S$. mansoni infection was later simply categorized as being either positive or negative.

\section{Urine sample processing using POC-CCA technique}

Additionally, each participating child was also asked to provide urine sample which was processed in the laboratory within $24 \mathrm{~h}$ for the qualitative presumptive detection of an active $S$. mansoni eggs by the commercially available POC-CCA technique, according to manufacturer's instructions (Rapid Medical Diagnostics, Pretoria) [25]. Two drops of urine (circa $80 \mu \mathrm{l})$ was transferred to the circular well of the test cassette and allowed to absorb entirely into the specimen pad, results were then read by two different technicians after $20 \mathrm{~min}$ after the assay had developed and any discrepancies examined by a third senior technician. The CCA tests were scored as negative, trace or positive (regardless of whether single or double band) based on formation and intensity of the control band [26, 27]. However, tests were considered invalid if an internal control band did not appear or if tests were not read after more than $25 \mathrm{~min}$ [1]. In those few cases, samples were re-run with a new cassette and scored as necessary. Tests were given a score of 'trace' if the control band was barely visible. In all our analysis herein, all 'trace' results were considered positive in agreement with van Dam et al. [26]. For quality control purposes, $10 \%$ of all urine samples were randomly reexamined by a senior technologist.
Both stool and urine samples were collected between 09:00 and 12:00 h each day of the survey. As part of the national school-based deworming programme, all participating children were treated with praziquantel for Schistosome infections according to WHO guidelines [24].

\section{Statistical analysis \\ Calculation of Kato-Katz parameters}

True positive (TP) was defined as the number of both Kato-Katz and POC-CCA positive samples, true negative (TN) was the number of both Kato-Katz and POC-CCA negative samples, false positives (FP) were the number of Kato-Katz positive samples and POC-CCA negative samples, and false negatives (FN) were the number of KatoKatz negative samples and POC-CCA positive samples. Therefore, the positive predictive values (PPV) were obtained using $T P /(T P+F P) x 100 \%$ whereas the negative predictive values (NPV) were obtained as $T N /(T N+F N)$ $x 100 \%$. Sensitivity $(\mathrm{Sn})$ was calculated as $T P /(T P+F N)$ $x 100 \%$ and specificity (Sp) was $T N /(T N+F P) x 100 \%$. Under the current study, the performance of Kato-Katz thick smear method was evaluated against the POC-CCA technique as the reference test 'gold standard'.

Kato-Katz performance was calculated and compared with the reference test, at $95 \%$ confidence intervals (CIs) for the following measures; Sn, Sp, PPV, NPV, positive likelihood ratio $\left(\mathrm{LR}^{+}\right)$, negative likelihood ratio $\left(\mathrm{LR}^{-}\right)$, and kappa score. Sensitivity and specificity were determined using $2 \times 2$ contingency tables and compared using McNemar's chi-square test. PPV and NPV were determined using the weighted generalized score chi-square test for paired data [28]. Exact binomial 95\%CI was calculated for each measure listed. Agreement between the two diagnostic methods was determined by calculating kappa statistics with 95\%CIs with kappa values interpreted according Landis and Koch classification [29].

The overall prevalence of $S$. mansoni infection from each diagnostic method was calculated at the school and county level and the $95 \% \mathrm{CI}$ obtained using binomial regression model.

All statistical analyses were carried out using STATA version 14.0 (STATA Corporation, College Station, TX, USA).

\section{Results}

Overall, 1899 children with mean age of 9.7 years (218 years) were surveyed in the pre-treatment survey, and 1878 children with mean age 9.4 years were surveyed during the post-treatment survey as shown in Table 1. All the pre-treatment surveys were conducted approximately 17 days before the treatment day and post-treatment surveys conducted 12-30 days after the treatment delivery. Information on sex was recorded for $98.8 \%$ of the children out of which $50.4 \%$ were male. The study did not necessarily survey same children during both pre- and post- 
Table 1 Number of schools and children examined by county

\begin{tabular}{llllll}
\hline County & \multicolumn{2}{l}{ Pre-treatment } & & \multicolumn{2}{l}{ Post-treatment } \\
\cline { 2 - 3 } & $\begin{array}{l}\text { Number of } \\
\text { schools }\end{array}$ & $\begin{array}{l}\text { Number of } \\
\text { children }\end{array}$ & & $\begin{array}{l}\text { Number of } \\
\text { schools }\end{array}$ & $\begin{array}{l}\text { Number of } \\
\text { children }\end{array}$ \\
\hline Bomet & 1 & 108 & 1 & 103 \\
Bungoma & 1 & 108 & 1 & 105 \\
Busia & 2 & 214 & 2 & 210 \\
Homa Bay & 1 & 108 & 1 & 102 \\
Kakamega & 2 & 212 & 2 & 213 \\
Kisii & 1 & 105 & 1 & 103 \\
Kisumu & 3 & 312 & 3 & 313 \\
Kwale & 3 & 317 & 3 & 316 \\
Mombasa & 1 & 98 & 1 & 108 \\
Narok & 2 & 215 & 2 & 214 \\
Taita & 1 & 102 & 1 & 91 \\
Taveta & & 1899 & 18 & 1878 \\
Overall & 18 & & & & \\
\hline
\end{tabular}

treatment surveys and those children absent on the day of the survey were not included in the study; moreover, children who did not provide both stool and urine samples were excluded from further analysis.

Table 2 compares the observed S. mansoni prevalence using the two techniques in overall and by county. The prevalence of $S$. mansoni infection was calculated with one Kato-Katz technique and compared with one POC-CCA technique. The observed prevalence using POC-CCA technique was $26.5 \%$ (95\% CI: 24.6-28.6) during pre-treatment and $21.4 \%$ (95\% CI: 19.6-23.4) during post-treatment compared to those observed when using Kato-Katz technique of 4.9\% (95\% CI: $4.0-5.9)$ and 1.5\% (95\%CI: $1.0-2.1)$ for pre- and post-treatment respectively. The observed prevalence for both pre- and post-treatment of $S$. mansoni infection using POC-CCA technique were significantly higher $\left(\chi^{2}=135.58, p<0.001\right)$ than those observed using Kato-Katz technique.

The number of children who tested positive or negative for each of the diagnostic methods is shown in Table 3, with the results showing that among the 1761 (92.7\%) samples examined during pre-treatment, 446 discrepancies were recorded (27 false positives and 419 false negatives), while only 370 discrepancies being recorded during post-treatment.

Taking POC-CCA as the gold standard, Kato-Katz significantly correctly identified only 80 out of 862 POCCCA - positive S. mansoni infections; $9.3 \% \mathrm{Sn},(95 \% \mathrm{CI}$ : 7.4-11.4; McNemar test $=782.0, p<0.001)$ and 2664 out of 2698 POC-CCA - negative samples; $98.7 \% \mathrm{Sp},(95 \%$ CI: 98.2-99.1; McNemar test $=34.0, p<0.001)$. The sensitivity of Kato-Katz was twice lower during posttreatment than pre-treatment (pre-treatment $\mathrm{Sn}=12.5 \%$, post-treatment $\mathrm{Sn}=5.2 \%, p<0.001)$. Overall, Kato-Katz resulted in a slight/poor detection of S. mansoni infection; $\mathrm{k}=0.11, p<0.001$, concordance $=77.1 \%$ (Table 4 ). In all those counties i.e. Bomet, Kisii, Kwale and Taita Taveta, where S. mansoni prevalence was zero during both pre- and post-treatment by Kato-Katz technique, sensitivity of Kato-Katz was also found to be zero.

Suppose otherwise we take Kato-Katz as the gold standard, the overall POC-CCA sensitivity was found to be $70.2 \%$ and specificity was $77.3 \%$, with higher

Table 2 Comparison of S. mansoni prevalence (\%) using POC-CCA and Kato-Katz techniques among school aged children

\begin{tabular}{|c|c|c|c|c|c|c|c|}
\hline \multirow[t]{2}{*}{ County } & \multicolumn{3}{|l|}{ Kato Katz } & \multicolumn{3}{|l|}{ POC-CCA } & \multirow{2}{*}{$\begin{array}{l}\text { Kappa } \\
\text { statistics } \\
\text { (Agreement } \\
\%)\end{array}$} \\
\hline & Pre-treatment (\%) & Post-treatment (\%) & RR (\%) & Pre-treatment (\%) & Post-treatment (\%) & RR (\%) & \\
\hline Overall & $4.9(4.0-5.9)$ & $1.5(1.0-2.1)$ & 69.8 & 26.5 (24.6-28.6) & $21.4(19.6-23.4)$ & 19.4 & $0.11(77.1 \%)$ \\
\hline Bomet & 0 & 0 & 0 & $61.9(53.3-71.9)$ & $54.9(46.0-65.5)$ & 11.3 & $0.00(42.1 \%)$ \\
\hline Bungoma & 0 & $2.0(0.5-7.7)$ & + & $8.6(4.6-16.0)$ & $14.6(9.1-23.2)$ & + & $0.06(88.8 \%)$ \\
\hline Busia & $28.7(23.2-35.5)$ & $2.9(1.3-6.4)$ & $89.9^{*}$ & $46.6(40.3-53.9)$ & $23.3(18.2-29.9)$ & $50.0^{*}$ & $0.28(71.4 \%)$ \\
\hline Homa Bay & 0 & $3.0(1.0-9.1)$ & + & $8.3(4.5-15.6)$ & $13.9(8.5-22.5)$ & + & $0.13(89.3 \%)$ \\
\hline Kakamega & $1.9(0.7-5.0)$ & $5.8(3.3-10.0)$ & + & $20.2(15.4-26.5)$ & $36.8(30.8-44.0)$ & + & $0.12(73.4 \%)$ \\
\hline Kisii & 0 & 0 & 0 & $12.1(7.1-20.6)$ & $15.0(9.4-23.9)$ & + & $0.00(86.1 \%)$ \\
\hline Kisumu & $7.1(4.7-10.6)$ & $1.3(0.5-3.5)$ & $81.4^{*}$ & $34.3(29.4-40.1)$ & $14.1(10.7-18.6)$ & $58.9^{*}$ & $0.15(77.4 \%)$ \\
\hline Kwale & 0 & 0 & 0 & $6.6(4.3-10.2)$ & $8.5(5.9-12.3)$ & + & $0.00(92.5 \%)$ \\
\hline Mombasa & $1.9(0.3-13.4)$ & 0 & 100 & $9.7(5.2-18.0)$ & $5.6(2.6-12.2)$ & 42.1 & $-0.01(90.3 \%)$ \\
\hline Narok & $0.9(0.2-3.7)$ & 0 & 100 & $47.4(41.1-54.6)$ & $42.9(36.7-50.1)$ & 9.5 & $0.01(55.2 \%)$ \\
\hline Taita Taveta & 0 & 0 & 0 & $19.2(12.8-28.8)$ & $2.4(0.6-9.3)$ & 87.7 & $0.00(88.5 \%)$ \\
\hline
\end{tabular}

$R R$ indicates relative reduction in prevalence

+indicates an increase in prevalence rather than reduction

*indicates a significant relative reduction in prevalence, $p<0.05$ 
Table 3 Comparative evaluation of the POC-CCA and the Kato-Katz parasitological examination for the diagnosis of S. mansoni infection

\begin{tabular}{|c|c|c|c|c|c|c|c|c|c|c|}
\hline \multirow[t]{3}{*}{ Diagnostic technique } & & \multicolumn{9}{|c|}{ Kato-Katz stool examination } \\
\hline & & \multicolumn{3}{|c|}{ Pre-treatment } & \multicolumn{3}{|c|}{ Post-treatment } & \multicolumn{3}{|l|}{ Overall } \\
\hline & & Positive & Negative & Total & Positive & Negative & Total & Positive & Negative & Total \\
\hline \multirow[t]{3}{*}{ POC-CCA urine examination } & Positive & 60 & 419 & 479 & 20 & 363 & 383 & 80 & 782 & 862 \\
\hline & Negative & 27 & 1255 & 1282 & 7 & 1409 & 1416 & 34 & 2664 & 2698 \\
\hline & Total & 87 & 1674 & 1761 & 27 & 1772 & 1799 & 114 & 3446 & 3560 \\
\hline
\end{tabular}

sensitivity and specificity noted during post-treatment, $(\mathrm{Sn}=74.1 \%, \mathrm{Sp}=79.5 \%$ respectively, and $p<0.001)$.

\section{Discussion}

National Schistosomiasis control programmes need diagnostic techniques which are sensitive, specific, rapid and easy to perform at point-of-care. Kato-Katz technique has long been the mainstay test in Schistosoma mansoni diagnosis in endemic areas by most epidemiological studies [2, 14]. However, recent studies have since documented its poor sensitivity in evaluating $S$. mansoni infection thus making it less useful especially in areas with lower rates of transmission [5-7]. The low sensitivity can be attributed to the relatively small stool sample examined, fluctuations in daily egg excretion and the heterogeneous distribution of eggs within the stool sample [30-32]. This study provides the first rigorous assessment of the performance of Kato-Katz technique in comparison to POC-CCA in a national Schistosomiasis control programme in selected areas with lower transmission rates in Kenya.

The number of children infected with $S$. mansoni as determined by POC-CCA assay of one urine sample was found to be significantly higher than those determined by duplicate Kato-Katz thick smears of one stool sample and indeed 5-fold higher during pre-treatment and 14-fold higher during posttreatment, this finding is in agreement with previous studies $[1,14,30,33,34]$. In fact, a recent systematic review by Kittur et al., [5] noted that whenever S. mansoni prevalence was above $50 \%$ by Kato-Katz then Kato-Katz and POC-CCA results yielded essentially the same prevalence. However, whenever the prevalence is below $50 \%$ by Kato-Katz then the
POC-CCA prevalence was between 1.5 and 6-fold higher and could increase further as prevalence by Kato-Katz decreased.

Out of the 862 POC-CCA positive samples, Kato-Katz classified $782(90.7 \%)$ as negative, a scenario which can be explained by the reasons mentioned above. On the other hand, of all the negative results by POC-CCA, Kato-Katz classified only 34 (1.3\%) as positive indicating a good specificity for Kato-Katz technique.

We noted that interpretation of the POC-CCA result band and inter-reader variability especially when the result is a 'trace' is an issue in the use of this technique, the same challenge had also been documented by other studies [35-37]. However, with better training in reading the result band, the challenge can be overcome. Other studies have suggested an addition of a comparison line on the assay to make reading of the result band easy and quick [34].

In comparison to one POC-CCA exam used as the gold standard, the stool-based Kato-Katz technique had extremely low sensitivity especially during post-treatment, but however had higher specificity both at pre- and posttreatment. In overall, the results demonstrated a significantly slight/poor inter-rater agreement between the two techniques; $\mathrm{k}=0.11, p<0.001$, agreement $=77.1 \%$. Our findings corroborate those of other studies done in Kenya [18], Uganda [15] and Ivory Coast [14] where POC-CCA tests detected $S$. mansoni infections in pre-school and school-aged at a higher sensitivity than the widely used Kato-Katz technique. Therefore, Kato-Katz and other direct diagnostic methods have inadequacies when it comes to accurate individual diagnosis which is further hampered by the fact that stools in young children are mostly diarrheic and renders the preparation of Kato-Katz thick smears difficult, hence challenging the accuracy of the diagnosis.

Table 4 Showing the performance measures of Kato-Katz by each survey round with POC-CCA as the gold standard

\begin{tabular}{|c|c|c|c|c|c|c|c|}
\hline Treatment round & Sensitivity \%(95\% Cl) & Specificity \%(95\% Cl) & $\mathrm{LR}^{+} \%(95 \% \mathrm{Cl})$ & $\mathrm{LR}^{-} \%(95 \% \mathrm{Cl})$ & PPV \%(95\% CI) & NPV \%(95\% Cl) & $\begin{array}{l}\text { Kappa index } \\
\text { (Agreement \%) }\end{array}$ \\
\hline Pre-treatment & $12.5(9.7-15.8)$ & 97.9 (97.0-98.6) & $6.0(3.8-9.3)$ & $0.9(0.9-0.9)$ & $69.0(58.1-78.5)$ & $75.0(72.8-77.0)$ & $0.14(74.7 \%)$ \\
\hline Post-treatment & $5.2(3.2-7.9)$ & 99.5 (99.0-99.8) & $10.6(4.5-24.8)$ & $1.0(0.9-1.0)$ & $74.1(53.7-88.9)$ & 79.5 (77.6-81.4) & $0.07(79.4 \%)$ \\
\hline $\begin{array}{l}\text { Overall } \\
\text { P-value** }\end{array}$ & $\begin{array}{l}9.3(7.4-11.4) \\
X^{2}{ }_{m}=782.0 \\
p<0.001\end{array}$ & $\begin{array}{l}98.7(98.2-99.1) \\
X^{2}{ }_{m}=34.0 \\
p<0.001\end{array}$ & $\begin{array}{l}7.4(5.0-10.92) \\
-\end{array}$ & $\begin{array}{l}0.9(0.9-0.9) \\
-\end{array}$ & $\begin{array}{l}70.2(60.9-78.4) \\
-\end{array}$ & $\begin{array}{l}77.3(75.9-78.7) \\
-\end{array}$ & $\begin{array}{l}0.11(77.1 \%) \\
Z=11.6 \\
p<0.001\end{array}$ \\
\hline
\end{tabular}

PPV positive predictive value, NPV negative predictive value, $L R^{+}$positive likelihood ratio, $L R^{-}$negative likelihood ratio

**Obtained from McNemar's chi-square $\left(\mathrm{X}^{2}{ }_{\mathrm{m}}\right)$ test (sensitivity \& specificity) / Weighted generalized score chi-square test (PPV \& NPV) 
Finally, the study showed that even a single urine-based POC-CCA test seemed to be a more appropriate and effective screening tool for $S$. mansoni evaluation compared to stool-based Kato-Katz smears in areas with low infection prevalence. The tool was more sensitive and up to 14-fold accurate than Kato-Katz method. Although, POCCCA test has known limitations like inter-reader variability in deciding a 'trace' result [30], more investigations can be conducted on how well the tool can distinguish between negative and 'trace' values. Even though the study found POC-CCA as a suitable alternative to Kato-Katz technique, it is known to provide limited intensity data and often no information on STHs infections [38], therefore we recommend its use for $S$. mansoni control programmes but with Kato-Katz for control programmes targeting both Schistosmiasis and STHs infections.

\section{Conclusions}

Most large-scale Schistosomiasis control programs are based on preventive chemotherapy which usually reduces the infection prevalence and intensity of Schistosomes [39]. The frequent treatment normally results in lowered endemicity which goes hand-in-hand with reduced accuracy of Kato-Katz technique [40]. Hence, the need of a more sensitive and specific diagnostic tool for examination of $S$. mansoni after extensive mass treatment cannot be over-emphasized. This current study found POC-CCA method as more effective, and sensitive and it was up to 14-fold accurate than Kato-Katz. It was easy to use and less time consuming.

\section{Abbreviations}

CAA: Cathodic anodic antigen; CIFF: Children's investment fund foundation; Cls: Confidence intervals; ECD: Early childhood development; EPG: Eggs per gram; FN: False negative; FP: False positive; KEMRI: Kenya medical research institute; KNSBDP: Kenya national school-based deworming programme; $L R^{-}$: Negative likelihood ratio; $\mathrm{LR}^{+}$: Positive likelihood ratio; M\&E: Monitoring and evaluation; MDA: Mass drug administration; NPV: Negative predictive values; NSBD: National school-based deworming; ODK: Open data kit; POCCCA: Point-of-care circulating cathodic antigen; PPV: Positive predictive values; Sn: Sensitivity; Sp: Specificity; STH: Soil-transmitted helminths; TN: True negative; TP: True positive; WHO: World health organization

\section{Acknowledgements \\ We are grateful to the Neglected Tropical Diseases Unit, Ministry of Health, Nairobi, Kenya and the County ministries of health and education for their unwavering support for this work. Additionally, we thank the school teachers and children who participated in this study for their support. Special thanks goes to all members of the study team and field personnel for their commitment towards this work. Finally, we want to thank CIFF for funding this work. CO is supported by CIFF through KEMRI-ESACIPAC as a Statistician, ES is also supported by CIFF through KEMRI-ESACIPAC as an Assistant Research Officer, and SMN and CM are supported by KEMRI as Chief Research Officers. This paper is published with the permission of the Director, KEMRI.}

\section{Funding}

The financial support for this research was provided by Children's Investment Fund Foundation (CIFF) through the grant number 208. The funders had no role in the study design, data collection and analysis, decision to publish or preparation of the manuscript.

\section{Availability of data and materials}

The datasets supporting the conclusions of this article are available upon request.

\section{Authors' contributions}

CO participated in the study design, data collection and analysis, and developed the draft manuscript. ES participated in data collection and laboratory procedures, SMN and CM participated in the study design and provided overall scientific guidance. All authors participated in interpretation of the findings, and read and approved the final manuscript.

\section{Ethics approval and consent to participate}

The study's proposal inclusive of the statement on ethical considerations (and methods of consent) was approved by the KEMRI's Scientific Steering Committee and the Ethics Review Committee (SSC No. 2206). Additional approval was provided by the county-level health and education authorities after they were appropriately briefed about the study. Since this was a cross-sectional study, at school-level, parents/guardians of all children in ECD class and those for classes 26 were called for a meeting in the school through the head teacher prior to the survey and the content of the survey explained to them. We used passive opt-out consent, where the parents/guardians were required only to return a signed form when they did not want their child to participate in the study. This kind of consenting was preferable due to the low risk nature of the study procedure and it ensured higher participation rate of the children and provided a more representative sample. Additionally, individual assent was obtained from each child before participation in the study.

\section{Competing interests}

The authors declare that they have no competing interests.

\section{Publisher's Note}

Springer Nature remains neutral with regard to jurisdictional claims in published maps and institutional affiliations.

Received: 18 December 2017 Accepted: 5 April 2018 Published online: 11 April 2018

\section{References}

1. Mwinzi PNM, Kittur N, Ochola E, Cooper PJ, Campbell CH, King CH, et al. Additional evaluation of the point-of-contact circulating cathodic antigen assay for Schistosoma mansoni infection. Front Public Health. 2015;3:48.

2. Katz N, Chaves A, Pellegrino J. A simple device for quantitative stool thick smear technique in schistosomiasis mansoni. Rev Soc Bras Med Trop. 1972;14:397-400.

3. WHO. Helminth control in school-age children. A guide for managers of control programmes. Geneva: World Health Organization; 2011.

4. de Vlas SJ, Gryseels B, van Oortmarssen GJ, Polderman AM, Habbema JDF. A pocket chart to estimate true Schistosoma mansoni prevalences. Parasitol Today. 1993;9:306-7.

5. Kittur N, Castleman JD, Campbell CH, King CH, Colley DG. Comparison of schistosoma mansoni prevalence and intensity of infection, as determined by the circulating cathodic antigen urine assay or by the kato-katz fecal assay: a systematic review. Am J Trop Med Hyg. 2016;94:605-10.

6. Shane HL, Verani JR, Abudho B, Montgomery SP, Blackstock AJ, Mwinzi PNM, et al. Evaluation of urine CCA assays for detection of Schistosoma mansoni infection in western Kenya. Bethony JM, editor. PLoS Negl Trop Dis. 2011;5:e951.

7. de Vlas SJ, Gryseels B. Underestimation of Schistosoma mansoni prevalences. Parasitol Today. 1992:8:274-7.

8. Hubbard A, Liang S, Maszle D, Qiu D, Gu X, Spear RC. Estimating the distribution of worm burden and egg excretion of Schistosoma japonicum by risk group in Sichuan Province, China. Parasitology. 2002;125:221-31.

9. Krauth SJ, Coulibaly JT, Knopp S, Traoré M, N'Goran EK, Utzinger J. An indepth analysis of a piece of shit: distribution of Schistosoma mansoni and hookworm eggs in human stool. PLoS Negl Trop Dis 2012;6:e1969.

10. Zhu HQ, Xu J, Zhu R, Cao CL, Bao ZP, Yu Q, et al. Comparison of the miracidium hatching test and modified Kato-Katz method for detecting Schistosoma japonicum in low prevalence areas of China. Southeast Asian J Trop Med Public Health. 2014;45:20-5.

11. Adriko M, Standley CJ, Tinkitina B, Tukahebwa EM, Fenwick A, Fleming FM et al. Evaluation of circulating cathodic antigen (CCA) urine-cassette assay as a survey tool for Schistosoma mansoni in different transmission settings within Bugiri District, Uganda. Acta Trop. 2014;136:50-7. 
12. Qian MB, Yap P, Yang YC, Liang H, Jiang ZH, Li W, et al. Accuracy of the Kato-Katz method and formalin-ether concentration technique for the diagnosis of Clonorchis sinensis, and implication for assessing drug efficacy. Parasit Vectors. 2013:6:314.

13. Sayasone S, Utzinger J, Akkhavong K, Odermatt P. Repeated stool sampling and use of multiple techniques enhance the sensitivity of helminth diagnosis: a cross-sectional survey in southern Lao People's Democratic Republic. Acta Trop. 2015;141:315-21.

14. Coulibaly JT, N'Gbesso YK, Knopp S, N'Guessan NA, Silué KD, van Dam GJ, et al. Accuracy of urine circulating cathodic antigen test for the diagnosis of Schistosoma mansoni in preschool-aged children before and after treatment. Liang S, editor. PLoS Negl Trop Dis. 2013;7:e2109.

15. Stothard JR, Sousa-Figuereido JC, Betson M, Adriko M, Arinaitwe M, Rowell $C$, et al. Schistosoma mansoni infections in young children: when are schistosome antigens in urine, eggs in stool and antibodies to eggs first detectable? PLoS Negl Trop Dis 2011;5:e938.

16. Coulibaly JT, Knopp S, N'Guessan NA, Silué KD, Fürst T, Lohourignon LK, et al. Accuracy of urine circulating cathodic antigen (CCA) test for Schistosoma mansoni diagnosis in different settings of Cote d'Ivoire. Lammie PJ, editor. PLoS Negl Trop Dis. 2011;5:e1384.

17. van Dam GJ, Bogitsh BJ, van Zeyl RJ, Rotmans JP, Deelder AM. Schistosoma mansoni: in vitro and in vivo excretion of CAA and CCA by developing schistosomula and adult worms. J Parasitol. 1996;82:557-64.

18. Verani JR, Abudho B, Montgomery SP, Mwinzi PNM, Shane HL, Butler SE, et al. Schistosomiasis among young children in Usoma, Kenya. Am J Trop Med Hyg. 2011:84:787-91.

19. Sousa-Figueiredo JC, Pleasant J, Day M, Betson M, Rollinson D, Montresor A, et al. Treatment of intestinal schistosomiasis in Ugandan preschool children: best diagnosis, treatment efficacy and side-effects, and an extended praziquantel dosing pole. Int Health. 2010;2:103-13.

20. Mwandawiro CS, Nikolay B, Kihara JH, Ozier O, Mukoko DA, Mwanje MT, et al. Monitoring and evaluating the impact of national schoolbased deworming in Kenya: study design and baseline results. Parasit Vectors. 2013;6:1.

21. Okoyo C, Nikolay B, Kihara J, Simiyu E, Garn JV, Freeman MC, et al. Monitoring the impact of a national school based deworming programme on soil-transmitted helminths in Kenya: the first three years, 2012 - 2014. Parasit Vectors. 2016;9:408.

22. CIFF. Kenya National Deworming Programme [Internet]. www.ciff.org. 2017 [cited 2018 Feb 18]. Available from: https://ciff.org/priorities/survive-thrive/ deworming/public-sector-delivery/kenya-national-deworming-programme/.

23. ODK. Open Data Kit [Internet]; 2009. p. 3-4. [cited 2018 Feb 18]. Available from: https://opendatakit.org/

24. WHO. Prevention and control of schistosomiasis and soil-transmitted helminthiasis: report of a WHO expert committee. In: WTS, editor. Report. Geneva: WHO; 2002. WHO technical report series.

25. Rapid Diagnostics. Rapid medical diagnostics [Internet]. 2015 [cited 2018 Feb 22]. Available from: http://www.rapid-diagnostics.com/.

26. Van Dam GJ, Wichers JH, Ferreira TMF, Ghati D, Van Amerongen A, Deelder AM. Diagnosis of schistosomiasis by reagent strip test for detection of circulating cathodic antigen diagnosis of schistosomiasis by reagent strip test for detection of circulating cathodic antigen. J Clin Microbiol. 2004:42:5458-61.

27. Kabatereine NB, Tukahebwa E, Kazibwe F, Namwangye H, Zaramba S, Brooker S, et al. Progress towards countrywide control of schistosomiasis and soil-transmitted helminthiasis in Uganda. Trans R Soc Trop Med Hyg. 2006;100:208-15.

28. Kosinski AS. A weighted generalized score statistic for comparison of predictive values of diagnostic tests. Stat Med. 2013;32:964-77.

29. Landis JR, Koch GG. An application of hierarchical kappa-type statistics in the assessment of majority agreement among multiple observers. Biometrics. 1977;33:363-74.

30. Casacuberta M, Kinunghi S, Vennervald BJ, Olsen A. Evaluation and optimization of the circulating cathodic antigen (POC-CCA) cassette test for detecting Schistosoma mansoni infection by using image analysis in school children in Mwanza region, Tanzania. Parasite Epidemiol Control. Elsevier. 2016;1:105-15

31. Berhe N, Medhin G, Erko B, Smith T, Gedamu S, Bereded D, et al. Variations in helminth faecal egg counts in Kato-Katz thick smears and their implications in assessing infection status with Schistosoma mansoni. Acta Trop. 2004;92:205-12.
32. Kongs A, Marks G, Verlé P, Van Der Stuyft P. The unreliability of the KatoKatz technique limits its usefulness for evaluating $S$. mansoni infections. Trop Med Int Health. 2001;6:163-9.

33. Erko B, Medhin G, Teklehaymanot T, Degarege A, Legesse M. Evaluation of urine-circulating cathodic antigen (urine-CCA) cassette test for the detection of Schistosoma mansoni infection in areas of moderate prevalence in Ethiopia. Trop Med Int Health. 2013;18:1029-35.

34. Colley DG, Binder S, Campbell C, King CH, Tchuenté LAT, N'Goran EK, et al. A five-country evaluation of a point-of-care circulating cathodic antigen urine assay for the prevalence of Schistosoma mansoni. Am J Trop Med Hyg. 2013;88:426-32.

35. Standley CJ, Lwambo NJS, Lange CN, Kariuki HC, Adriko M, Stothard JR. Performance of circulating cathodic antigen (CCA) urine-dipsticks for rapid detection of intestinal schistosomiasis in schoolchildren from shoreline communities of Lake Victoria. Parasit Vectors. 2010:3:7.

36. Stothard JR, Kabatereine NB, Tukahebwa EM, Kazibwe F, Rollinson D, Mathieson W, et al. Use of circulating cathodic antigen (CCA) dipsticks for detection of intestinal and urinary schistosomiasis. Acta Trop. 2006;97:219-28.

37. Stothard JR, Sousa-Figueiredo JC, Standley C, Van Dam GJ, Knopp S, Utzinger J, et al. An evaluation of urine-CCA strip test and fingerprick blood SEA-ELISA for detection of urinary schistosomiasis in schoolchildren in Zanzibar. Acta Trop. 2009;111:64-70.

38. Lamberton PHL, Kabatereine NB, Oguttu DW, Fenwick A, Webster JP. Sensitivity and specificity of multiple Kato-Katz thick smears and a circulating cathodic antigen test for Schistosoma mansoni diagnosis preand post-repeated-praziquantel treatment. Knopp S, editor. PLoS Negl Trop Dis. Public Library of Science. 2014;8:e3139.

39. Fenwick A, Webster JP, Bosque-Oliva E, Blair L, Fleming FM, Zhang Y, et al. The schistosomiasis control initiative (SCI): rationale, development and implementation from 2002-2008. Parasitology. 2009;136:1719.

40. Knopp S, Stothard JR, Rollinson D, Mohammed KA, Khamis IS, Marti H, et al. From morbidity control to transmission control: time to change tactics against helminths on Unguja Island, Zanzibar. Acta Trop. Elsevier. 2013;128:412-22.

\section{Ready to submit your research? Choose BMC and benefit from:}

- fast, convenient online submission

- thorough peer review by experienced researchers in your field

- rapid publication on acceptance

- support for research data, including large and complex data types

- gold Open Access which fosters wider collaboration and increased citations

- maximum visibility for your research: over $100 \mathrm{M}$ website views per year

At BMC, research is always in progress.

Learn more biomedcentral.com/submissions 